\title{
Spatial Distribution of Cytoskeletal Mechanical Properties in Vascular Smooth Muscle Cells
}

\author{
Qifeng Jiang1 ${ }^{*}$, Ping Chen², Jinhe Li ${ }^{2}$ \\ ${ }^{1}$ Department of Biochemistry and Molecular Biology, Chongqing Medical University, Chongqing, China \\ ${ }^{2}$ Department of Medical Information, Chongqing Medical University, Chongqing, China \\ Email: ${ }^{\text {jiqf1008@aliyun.com }}$
}

Received 27 April 2015; accepted 23 May 2015; published 27 May 2015

Copyright (C) 2015 by authors and Scientific Research Publishing Inc.

This work is licensed under the Creative Commons Attribution International License (CC BY). http://creativecommons.org/licenses/by/4.0/

c) (†) Open Access

\section{Abstract}

The mechanical properties on a whole basis of cell are extensively studied whereas regional mechanical properties of the cytoskeleton (CSK) have not been characterized. To fill this gap, here we provide detailed intracellular maps of regional CSK stiffness and traction forces, as well as the structural rearrangement in vascular smooth muscle cells (VSMCs). We used micropatterning to restrict VSMC to a single island. Regional cell stiffness was measured with optical magnetic twisting cytometry and traction force microscopy was used to measure regional traction forces. Finally, we used F-actin staining to measure the regional F-actin distribution. From traction and cell height data we computed the prestress within a cell using finite element methods. Compared with the cell center, cell edges were systematically stiffer, more fluid-like and supported higher traction forces, and at the same time had slower remodeling dynamics. These findings provide the most detailed characterization to date concerning regional mechanical properties of the intact cytoskeleton.

\section{Keywords}

\section{Cell Stiffness, Traction Forces, Cytoskeleton, Prestress}

\section{Introduction}

Mechanical properties of the cytoskeleton (CSK) play critical role in cell spreading [1], crawling [2] [3], invasion [4], division [5], pattern formation [6], and gene expression [7]. For most of these cellular activities, cells need to tune CSK material properties not only globally at the whole cell level but also regionally within the sin-

${ }^{*}$ Corresponding author.

How to cite this paper: Jiang, Q.F., Chen, P. and Li, J.H. (2015) Spatial Distribution of Cytoskeletal Mechanical Properties in Vascular Smooth Muscle Cells. J. Biomedical Science and Engineering, 8, 350-356. 
gle cell [8]. While structural studies abound, systematic regional variations of CSK material properties and dynamics within a single cell have yet to be defined in any detail [9]. In the single micropatterned cell, the cell corner, in particular, is known to correspond to the region of greatest focal adhesion density, highest traction forces [10], and most active lamella extension [11] [12], but how these regional features are related to local material properties of the CSK remains unclear.

Here we used micropatterned substrates to restrict the human vascular smooth muscle cell to a single extracellular matrix island. Mircopatterning afforded several experimental advantages, which could create well-defined cell corners with no appreciable variation in cell spreading or cell shape. For that reason, responses could be averaged across an ensemble of cells with virtually identical geometries, and statistical power could be amplified further by taking advantage of the 8-fold symmetry condition implied by single cell geometry and the associated rectilinear coordinate system onto which all data could be unambiguously mapped.

We used confocal-microscopy to measure regional variations in filamentous actin [10] [11], and fouriertransform traction microscopy (FTTM) to measure regional variations in tractions that cell exerts on its elastic substrate. In addition, we used forced tracer displacements (optical magnetic twisting cytometry, OMTC) to measure regional variations in cell storage modulus g' and loss modulus g” [13]. As described below, g' and g” were measured as a function of the frequency $f$ at which forced tracer displacements were imposed [14]. Finally, from these observations together with Newton's third law and finite element methods (FEM), we were able to compute for the first time the distribution of the local tensional prestress.

Using these approaches we found systematic distributional patterns of cell mechanical properties that were not accounted for by systematic variations in regional cell height; compared with the center or the edges, the cell corner was found to have higher tractions, higher stiffness, and smaller rates of remodeling dynamics. Moreover, in the cell corners the F-actin network was more dense and under greater tension.

\section{Material and Methods}

Cell culture: The primary human aortic smooth muscle cells (HASMCs) were obtained from Sciencell, San Diego, US. Cells were cultured in smooth muscle cell complete medium (Sciencell, San Diego, US) and placed in a humidified incubator at $37^{\circ} \mathrm{C}$ and $5 \% \mathrm{CO}_{2}$. Cells were passaged while $80 \%$ confluence. Cells between passage 3 - 7 were used for all experiments, and they were serum deprived for $24 \mathrm{~h}$ before being tested.

Micropatterning: Polydimethylsiloxane (PDMS) membranes with $50 \times 50 \mu \mathrm{m}$ single holes were fabricated using conventional micromachining procedures and put onto the center of a glass-bottom well or on top of the acryl amide gel surface, then we coated the open surfaces with collagen (Vitrogen, Cohesion, CA). $5 \mu \mathrm{g} / \mathrm{ml}$ and $100 \mu \mathrm{g} / \mathrm{ml}$ collagen solution were used to coat glass and gel surfaces, respectively. After incubation at $4^{\circ} \mathrm{C}$ overnight, we lifted off the membrane and blocked the uncoated area with 1\% bovine serum albumin (A9647, Sigma, MO). Each cell was micropatterned onto a single $50 \mu \mathrm{m}$ single which was chosen based on the average area of isolated human airway smooth muscle cell. We plated serum-deprived cells ( 1500 cells/well) sparsely, washed 2 times with fresh medium after 1 hour and incubated overnight before starting experiments. Single cells that were assumed in single shape were used for measurements. To minimize variation, we aligned the single cells in parallel to the measuring area.

Optical magnetic twisting cytometry (OMTC) and experimental procedure: As a classical method of cell stiffness measurement, the OMTC technique is well documented [13]. First, the RGD-coated magnetic beads were added onto cells seeded on the gel substrates and incubated for $30 \mathrm{~min}$ to make sure the beads bind to receptors of the cell membrane. Second, the dish was fixed onto a microscope stage custom fitted with the bead twisting setup. The beads were magnetized horizontally and then twisted in an oscillatory magnetic field with a frequency of $0.75 \mathrm{~Hz}$. From the ratio of the applied mechanical torque to the measured lateral bead displacement, we could calculate the complex elastic modulus which is given by $G^{*}=G^{\prime}+j G^{\prime}$, where $G^{\prime}$ is the elastic modulus, or cell stiffness, which has units of Pascal per nanometer, $G^{\prime \prime}$ is the loss modulus which has units of Pascal per nanometer, and $j$ is the unit imaginary number. $G$ ' was measured in 6 wells for each experimental condition. Data were reported as medians of the bead populations (>300 beads on a hundreds of cells per experimental condition).

Fourier-transform traction microscopy: Bright field and fluorescent images of the micropatterned cell on a gel were recorded. After trypsinizing the cell, another fluorescent image was taken to calculate the displacement field generated by cell. Using a bright field image and two fluorescent images before and after trypsinizing cell, 
we determined the displacement field of the gel. In brief, fluorescent images were divided into a number of small window areas. The displacement field between a pair of images was obtained by identifying the coordinates of the peak of the cross-correlation function of each pair of window areas. The constrained traction field was calculated from the displacement field and cell boundary which was drawn to be a $50 \mu \mathrm{m}$ single, implementing the solution described by Butler et al. [15]

Confocal microscopy: Cells were washed 2 times with warm PBS and then fixed with 4\% paraformaldehyde. Cells were then permeablized using $0.03 \%$ Triton $\mathrm{x}-100$, incubated with $1 \%$ BSA to minimize non-specific binding, and then incubated with rhodamine phalloidin (1:20, Molecular Probes, OR) and Sytox green nucleic acid stain (1:100,000, Molecular Probes, OR). The structure of F-actin and the nucleus were scanned using confocal microscope (Leica TCSNT, Leica Microsystems, IL) with fixed step size $(0.2 \mu \mathrm{m})$. The images were stacked over whole cell thickness.

Statistical analysis: Data in the octant were divided into two groups based on the position; center (Ce, $\mathrm{x}<$ $12.5 \mu \mathrm{m})$, corner $(\mathrm{Co}, \mathrm{y}>12.5 \mu \mathrm{m})$. Statistical analyses were performed using unpaired t-test assuming unequal variance. If a variable followed log-normal distribution, we analyzed statistics after applying logarithm to the variable. Statistical significance was assumed at the level of p less than 0.05 .

\section{Results}

\subsection{Cell Stiffness}

Cell stiffness, which is generated by interplay of cytoskeleton and extracellular matrix, is a critical cell mechanical property in normal cell functions and increasingly associated with disease states. Experimentally, cell internal stiffness can be assessed by measuring the magnetic force-induced displacement of RGD-coated beads attached at the dorsal cell surface. Two representative single cells with bound RGD-coated beads are shown in Figure 1(a). When we pooled data from a total of 193 different micropatterned cells, we obtained mechanical data from a total of 462 beads. As a crude first analysis, we segregated beads into two groups: center and corner of the cell (Figure 1(b)). When we plotted the geometric mean of elastic shear modulus (g') as a function of frequency, the modulus increased as a weak power law in both regions (Figure 2(a)). The corner was approximately twice as stiff as the center. The loss modulus showed the regional differences comparable to the storage modulus (Figure 2(b)).

\subsection{Traction Forces Distribution}

The traction force is the net force per unit area transmitted from the adherent cell to the substrate, and must be balanced by the prestress generated by cytoskeleton in the cell body. The intensity of traction force is not only involved in cellar morphology but also in cell proliferation and migration. For a micropatterned cell on elastic gel, cell-induced displacements of the elastic substrate were biggest at the cell corners, and traction was concentrated in these corners (Figure 3(a)). There was minimal traction in cell center and the directions of traction vectors were mostly centripetal.

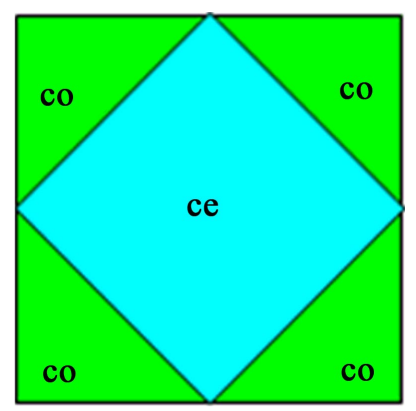

(a)

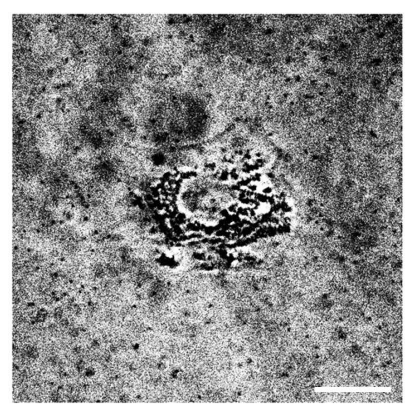

(b)

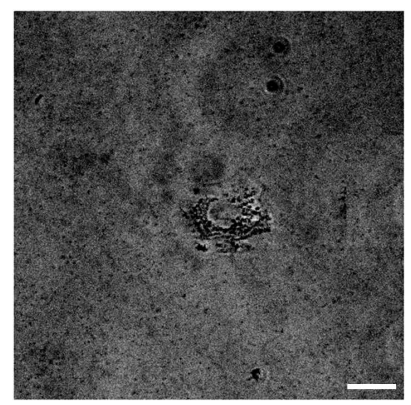

Figure 1. (a) All the beads were mapped onto an octant of the single. The cellular region was further divided into 2 regions; Ce (center) and Co (corner); (b) Bright field (left) and phase contrast (right) images of micropatterned human vascular smooth muscle cells with magnetic beads. Scale bar: $25 \mu \mathrm{m}$. 


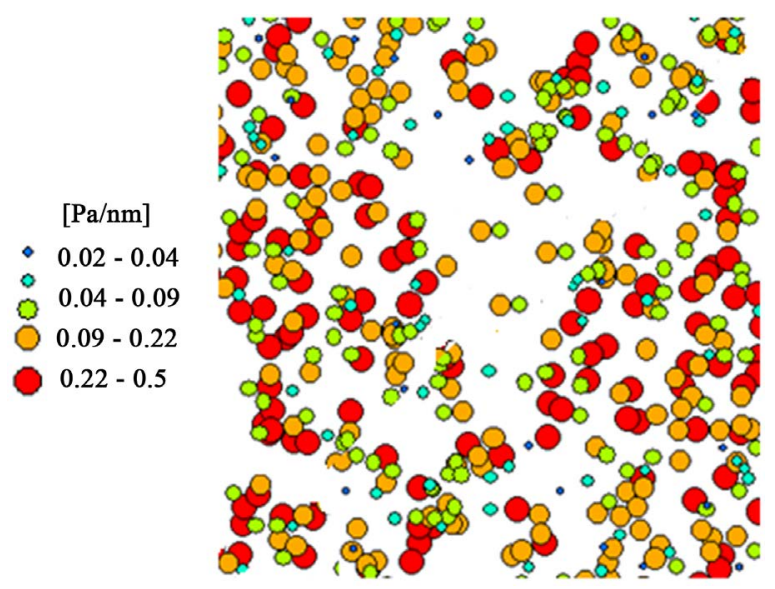

(a)

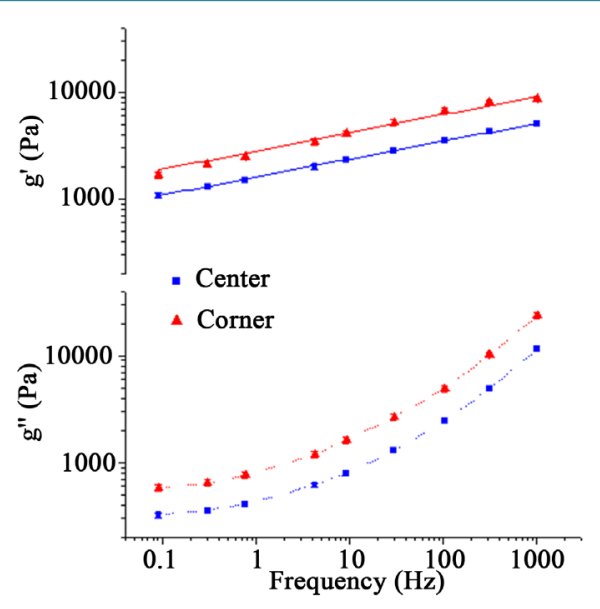

(b)

Figure 2. (a) Local shear modulus (g $[\mathrm{Pa} / \mathrm{nm}]$ ) measured by OMTC were transformed onto the octant of the single cell; (b) Average storage (g') and loss (g') modulus of each regions show a power law dynamics as a function of frequency. The data presented are geomean $\pm \varepsilon_{\text {log }}{ }^{*}$ geomean where geomean is geometrical mean and $\varepsilon_{\text {log }}$ is standard error in log space. g' and g' 'in each region were significantly different with each other at all frequency except for the g' at $1 \mathrm{kHz}$.

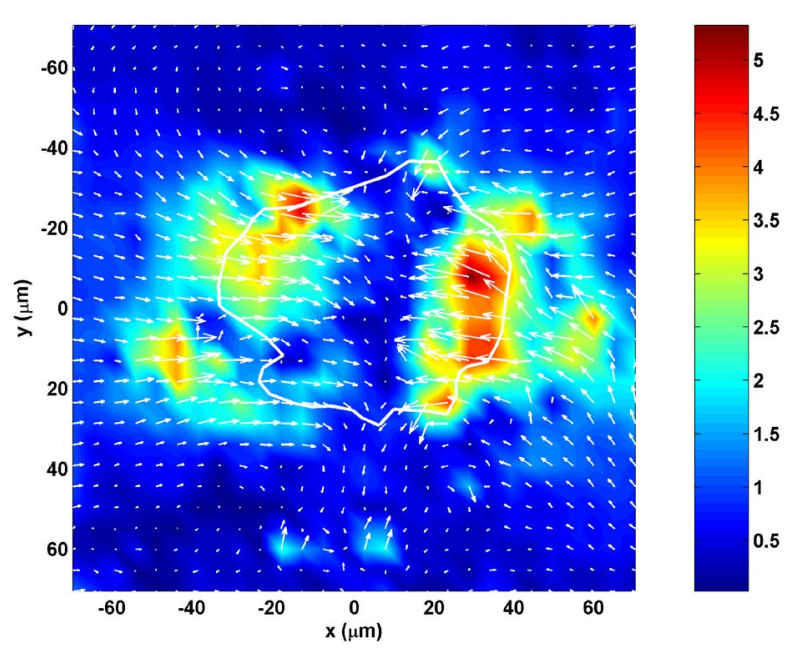

(a)

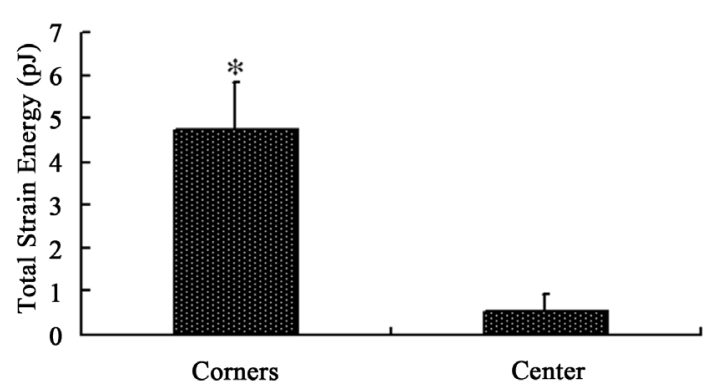

(b)

Figure 3. (a) Distribution of average traction (mean, $n=13$ ). Color of the map denote the magnitude and arrows do the magnitude and direction of the tractions; (b) Results of relative traction forces (pJ) in cell center and corners: the relative traction forces in cell corners were nearly 10 -fold more than cell center.

\subsection{The Distribution of F-Actin}

To further investigate the role of F-actin in regional heterogeneity, we stained F-actin with rhodamine phalloidin and measured regional distribution of the amount of F-actin. F-actin was highly concentrated around the cell corners (Figure 4(a)), and the relative concentration of the F-actin in cell corners was almost 8-fold more than cell center (Figure 4(b)).

\subsection{The Distribution of Prestress}

The distribution of cytoskeletal internal stress was calculated using local cell height, the distribution of traction forces at the cell base, and Newton's third law. As a measure of the local prestress we used the maximal principal stress (Figure 5). The magnitude of prestress was on the order of $\mathrm{kPa}$. The prestress was largest in the corners and decreased along the diagonal line toward cell center. The direction of prestress along the diagonal 


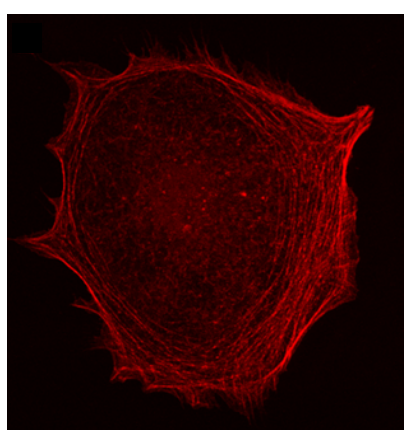

(a)

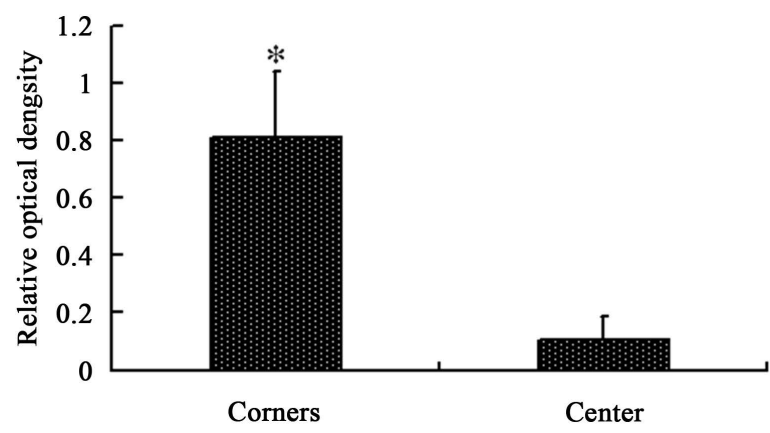

(b)

Figure 4. (a) Confocol image of F-actin in a single cell (b) Mean optical density of F-actin in a single cell $(n=36)$.

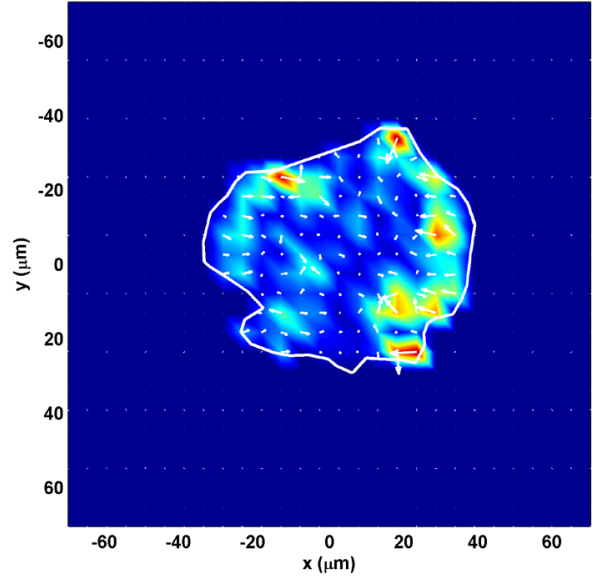

(a)
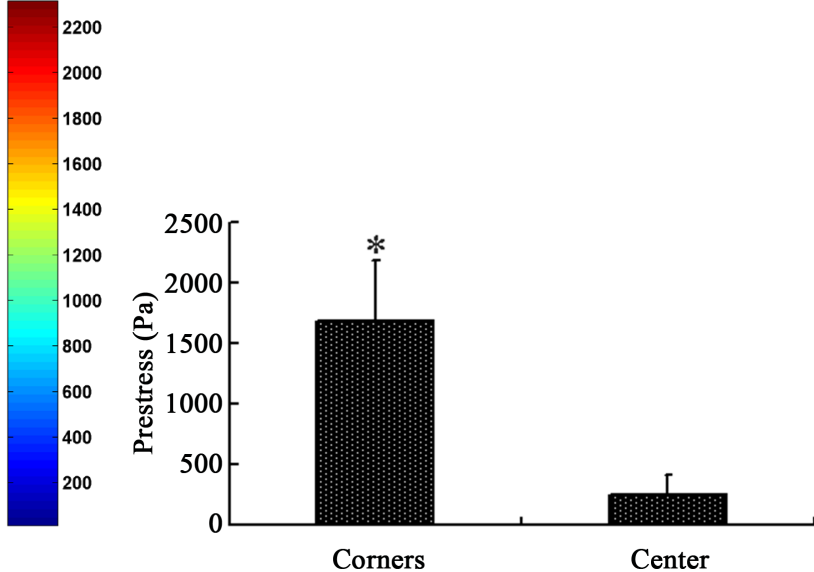

(b)

Figure 5. (a) Distribution of prestress within a single cell; (b) Results of relative prestress (Pa) in cell center and corners: the relative prestress in cell corners were nearly 8-fold more than cell center.

line was centripetal and parallel to the direction of traction. The prestress along the edge of single cell, however, was parallel to the cell boundary and perpendicular to the prestress direction.

\section{Discussion}

Here we have examined in the micropatterned single cell systematic regional variations of CSK mechanical properties. Compared with cell center, cell corners were systematically stiffer, had slower remodeling dynamics, supported higher traction forces, had higher level of prestress, and had higher concentration of F-actin filaments. These data provide the most comprehensive characterization to date of regional variations of mechanical properties in the intact cytoskeleton.

Cell stiffness, generated by interplay of cytoskeleton and extracellular matrix, is interpreted as the deformability of cells or resistance to morphological change. Many reports have shown that cell stiffness is essential for regulation of cell morphology, adhesion, spreading and motility [16]-[18]. When we compared the stiffness between cell corners and center in HASMCs, we found that stiffness was significantly decreased in cell center compared with corners (Figure 2).

Using cells adherent upon a static elastic substrate, Dembo and Wang [19] were the first to show that the traction forces that a cell exerts on its surroundings can be mapped quantitatively from knowledge of the displacement field induced by the cell in a passive flexible substrate on which the cell is adherent. In that scheme, calculation of the traction field from the measured displacement field is computationally intensive, however. To relieve this computational bottleneck, we used Fourier Transform Traction Microscopy (FTTM), a computationally efficient method for computing the traction field given the displacement field [19]. FTTM is fundamentally 
different from Dembo's approach in several respects, but is no less rigorous. Our data showed that cell corners presented much higher traction forces than cell center (Figure 3), it might be because cell corners are the major source of the power for cell migration and invasion.

Since the dynamics of traction forces and stiffness showed so distinct difference between cell center and corners, we furthered our studies to probe the potential mechanism underlying in this phenomenon. The common resource to generate traction force and stiffness is the cytoskeleton frameworks. It has been well addressed that both internal and external physical forces can act through the cytoskeleton to affect local mechanical properties of cells. Our results provided direct evidence that distribution of cell stiffness and traction forces was quite parallel with cytoskeleton distribution. F-actin was highly concentrated around the cell corners (Figure 4(a)), and the relative concentration of the F-actin in cell corners was almost 8-fold more than cell center (Figure 4(b)).

Gardel et al. found that the shear modulus of constituted actin gel cross-linked with filamin depended only on the prestress but not on the concentrations of actin or cross-linker if the prestress was applied onto the gel [20] [21]. In this paper, we present the internal stress distribution within a cell for the first time (Figure 5) and show that the local prestress is more related with the acto-myosin activity level than the shear modulus (Figure 4). When we define the local prestress from the stress distribution in a cell, the definition was not straightforward to make because the traditional definition of prestress was average stress acting on a cross-sectional surface of a cell to balance traction on the cell bottom. We used the maximal principal stress as a measure of prestress because we assumed that the prestress would be the most dominant stress within CSK network and the maximal principal stress was the closest equivalent one in a numerical model.

\section{Conclusion}

In conclusion, we found a cell has very heterogeneous distributions of CSK mechanical properties; corners are more fluid-like and stiffer and support more traction than the other regions. Here we argue that the origins of these heterogeneities are the regional distribution of acto-myosin activities. But further experimental studies are required to explore this fundamental question.

\section{Acknowledgements}

We would like to thank Prof. S.-X. Cai, Department of Bioengineering, Chongqing University for suggestion. We would like to thank members of the Department of Biochemistry and Molecular Biology, Chongqing Medical University for technical supports. This study was supported by NNSFC (National Natural Science Foundation of China, No. 31201047 to Jiang), by SRFDP (Research Fund for the Doctoral Program of Higher Education, No. 20125503120018 to Jiang), by the open fund of Key Laboratory of Ministry of Education (No. CQKLBST-2012-005 to Jiang).

\section{References}

[1] Chen, C.S., et al. (1997) Geometric Control of Cell Life and Death. Science, 276, 1425-1428. http://dx.doi.org/10.1126/science.276.5317.1425

[2] Small, J.V. and Resch, G.P. (2005) The Comings and Goings of Actin: Coupling Protrusion and Retraction in Cell Motility. Current Opinion in Cell Biology, 17, 517-523. http://dx.doi.org/10.1016/j.ceb.2005.08.004

[3] Li, S., Guan, J.L. and Chien, S. (2005) Biochemistry and Biomechanics of Cell Motility. Annual Review of Biomedical Engineering, 7, 105-150. http://dx.doi.org/10.1146/annurev.bioeng.7.060804.100340

[4] Yamazaki, D., Kurisu, S. and Takenawa, T. (2005) Regulation of Cancer Cell Motility through Actin Reorganization. Cancer Science, 96, 379-386. http://dx.doi.org/10.1111/j.1349-7006.2005.00062.x

[5] Matzke, R., Jacobson, K. and Radmacher, M. (2001) Direct, High-Resolution Measurement of Furrow Stiffening during Division of Adherent Cells. Nature Cell Biology, 3, 607-610. http://dx.doi.org/10.1038/35078583

[6] Ingber, D.E. (2006) Mechanical Control of Tissue Morphogenesis during Embryological Development. The International Journal of Developmental Biology, 50, 255-266. http://dx.doi.org/10.1387/ijdb.052044di

[7] Fredberg, J.J. and Kamm, R.D. (2006) Stress Transmission in the Lung: Pathways from Organ to Molecule. Annual Review of Physiology, 68, 507-541. http://dx.doi.org/10.1146/annurev.physiol.68.072304.114110

[8] Ingber, D.E. (2003) Mechanosensation through Integrins: Cells Act Locally But Think Globally. Proceedings of the National Academy of Sciences of the United States of America, 100, 1472-1474. 
http://dx.doi.org/10.1073/pnas.0530201100

[9] Heidemann, S.R. and Wirtz, D. (2004) Towards a Regional Approach to Cell Mechanics. Trends in Cell Biology, 14, 160-166. http://dx.doi.org/10.1016/j.tcb.2004.02.003

[10] Parker, K.K., et al. (2002) Directional Control of Lamellipodia Extension by Constraining Cell Shape and Orienting Cell Tractional Forces. The FASEB Journal, 16, 1195-1204. http://dx.doi.org/10.1096/fj.02-0038com

[11] Brock, A., Chang, E., Ho, C.-C., LeDuc, P., Jiang, X.Y., Whitesides, G.M. and Ingber, D.E. (2003) Geometric Determinants of Directional Cell Motility Revealed Using Microcontact Printing. Langmuir, 19, 1611-1617. http://dx.doi.org/10.1021/la026394k

[12] Kidoaki, S. and Matsuda, T. (2007) Shape-Engineered Vascular Endothelial Cells: Nitric Oxide Production, Cell Elasticity, and Actin Cytoskeletal Features. Journal of Biomedical Materials Research Part A, 81A, 728-735. http://dx.doi.org/10.1002/jbm.a.31112

[13] Trepat, X., Deng, L.H., An, S.S., Navajas, D., Tschumperlin, D.J., Gerthoffer, W.T., et al. (2007) Universal Physical Responses to Stretch in the Living Cell. Nature, 447, 592-595. http://dx.doi.org/10.1038/nature05824

[14] Fabry, B., Maksym, G.N., Shore, S.A., Moore, P.E., Panettieri Jr., R.A., Butler, J.P. and Fredberg, J.J. (2001) Selected Contribution: Time Course and Heterogeneity of Contractile Responses in Cultured Human Airway Smooth Muscle Cells. Journal of Applied Physiology, 91, 986-994.

[15] Butler, J.P., Tolic-Norrelykke, I.M., Fabry, B. and Fredberg, J.J. (2002) Traction Fields, Moments, and Strain Energy That Cells Exert on Their Surroundings. AJP: Cell Physiology, 282, C595-C605. http://dx.doi.org/10.1152/ajpcell.00270.2001

[16] Tee, S.Y., Fu, J., Chen, C.S. and Janmey, P.A. (2011) Cell Shape and Substrate Rigidity Both Regulate Cell Stiffness. Biophysical Journal, 100, L25-L27.

[17] Solon, J., Levental, I., Sengupta, K., Georges, P.C. and Janmey, P.A. (2007) Fibroblast Adaptation and Stiffness Matching to Soft Elastic Substrates. Biophysical Journal, 93, 4453-4461. http://dx.doi.org/10.1529/biophysj.106.101386

[18] Lu, Y.B., Iandiev, I., Hollborn, M., Kirber, N., Ulbricht, E., Hirrlinger, P.G., et al. (2011) Reactive Glial Cells: Increased Stiffness Correlates with Increased Intermediate Filament Expression. The FASEB Journal, 25, 624-631.

[19] Dembo, M. and Wang, Y.L. (1999) Stresses at the Cell-to-Substrate Interface during Locomotion of Fibroblasts. Biophysical Journal, 76, 2307-2316. http://dx.doi.org/10.1016/S0006-3495(99)77386-8

[20] Gardel, M.L., Shin, J.H., MacKintosh, F.C., Mahadevan, L., Matsudaira, P. and Weitz, D.A. (2004) Elastic Behavior of Cross-Linked and Bundled Actin Networks. Science, 304, 1301-1305. http://dx.doi.org/10.1126/science.1095087

[21] Gardel, M.L., Nakamura, F., Hartwig, J.H., Crocker, J.C., Stossel, T.P. and Weitz, D.A. (2006) Prestressed F-Actin Networks Cross-Linked by Hinged Filamins Replicate Mechanical Properties of Cells. Proceedings of the National Academy of Sciences of the United States of America, 103, 1762-1767. http://dx.doi.org/10.1073/pnas.0504777103 